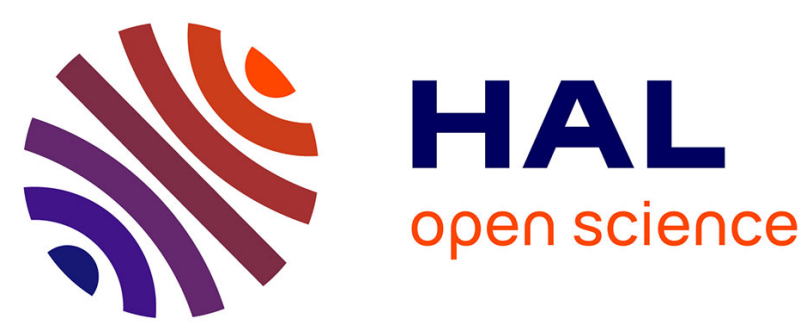

\title{
Auction-based Agent Negotiation in Cognitive Radio Ad Hoc Networks
}

Asma Amraoui, Badr Benmammar, Francine Krief, Fethi Tarik Bendimerad

\section{To cite this version:}

Asma Amraoui, Badr Benmammar, Francine Krief, Fethi Tarik Bendimerad. Auction-based Agent Negotiation in Cognitive Radio Ad Hoc Networks. ADHOCNETS 2012: 4th International Conference on Ad Hoc Networks, Oct 2012, Paris, France. hal-00730675v2

\section{HAL Id: hal-00730675 \\ https://hal.inria.fr/hal-00730675v2}

Submitted on 16 Mar 2013

HAL is a multi-disciplinary open access archive for the deposit and dissemination of scientific research documents, whether they are published or not. The documents may come from teaching and research institutions in France or abroad, or from public or private research centers.
L'archive ouverte pluridisciplinaire HAL, est destinée au dépôt et à la diffusion de documents scientifiques de niveau recherche, publiés ou non, émanant des établissements d'enseignement et de recherche français ou étrangers, des laboratoires publics ou privés. 


\title{
Auction-Based Agent Negotiation in Cognitive Radio Ad Hoc Networks
}

\author{
Asma Amraoui ${ }^{1}$, Badr Benmammar ${ }^{1}$, Francine Krief $^{2}$, and Fethi Tarik Bendimerad ${ }^{1}$ \\ ${ }^{1}$ LTT Laboratory, University of Tlemcen, Algeria \\ ${ }^{2}$ LaBRI Laboratory Bordeaux 1 University, Talence, France \\ \{amraoui.asma, badr. benmammar, ftbendimerad\} @gmail.com, \\ krief@labri.fr
}

\begin{abstract}
The explosive growth of wireless services in recent years illustrates the growing demand for communications, so the spectrum becomes more congested. We know that static spectrum allocation is a major problem in wireless networks. Generally, these allocations lead to an inefficient use of spectrum. To solve the problem of congestion, cognitive radio networks use dynamic spectrum access. In this paper, we use a technique based on auctions theory known for its simplicity and facilitates the allocation of scarce resources.
\end{abstract}

Keywords: Cognitive radio, multi agent systems, auctions theory, dynamic spectrum access.

\section{Introduction}

The Cognitive Radio (CR) is a form of wireless communication in which a transmitter / receiver can detect intelligently communication channels which are in use and those which are not and can move to unused channels. This optimizes the use of available radio frequency spectrum while minimizing interference with other users.

The principle of CR requires an alternative management of spectrum: a user called "secondary" may at any time access to the free channels, i.e., not occupied by the user called "primary" who possess a license on this band. The secondary user (SU) will give way the channel after completing the service or if a primary user (PU) has shown an inclination to login.

To make CR systems practical, CR networks must be able to coexist; this may cause interference to other users. To deal with this problem, the idea of cooperation between users to sense and share spectrum without causing interference is introduced.

The cooperative resolution of problems plays a predominant role in research in DAI $^{1}$ (Distributed Artificial Intelligence). A relatively complex area of research derived from the DAI is Multi Agent Systems (MAS). The topic MAS focuses on the study of collective behavior and the distribution of intelligence on autonomous agents able to organize themselves and interact to solve problems.

\footnotetext{
${ }^{1}$ Unlike Artificial Intelligence (AI) which models the intelligent behavior of a single agent, the DAI is concerned with intelligent behaviors that result from the cooperative activity of several agents.

J. Zheng et al. (Eds.): Adhocnets 2012, LNICST 111, pp. 119-134, 2013.

(C) Institute for Computer Sciences, Social Informatics and Telecommunications Engineering 2013
} 
Cooperation can be considered as an attitude adopted by agents which decide to work together. In the case of CR, before cooperation a step of negotiation is needed because there are many users who want to satisfy their needs. Negotiation plays a fundamental role in the cooperation activities by enabling people to resolve conflicts that could put in danger the cooperative behavior.

In this paper, we start by giving an overview on MAS and then discuss their use in the CR. We will establish a state of the art on the use of auctions in CR networks and then give the network topology and the negotiation algorithms that we propose

\section{Multi Agent Systems}

\subsection{Agent}

The concept of agent is used in several disciplines, so there are few compromises when it is question about defining the term "agent". However, one of the most known definitions which is considered as one of the firsts is that of [1]:

"An agent is an autonomous entity, real or abstract, which can act on itself and its environment, which in a multi agent environment can communicate with other agents, and whose behavior is the consequence of its observations, knowledge and interactions with other agents."

\subsection{Multi Agent Systems}

MAS are particularly suitable for reactive and robust solutions to complex problems for which there is no centralized control [2] [3]. Indeed, the MAS is a group of agents where each agent has one or more basic skills. The goal is to make work together agents to solve a problem or perform a specific task. Somehow, we distribute intelligence; each autonomous agent has only a local vision of the problem or an elementary task of a job.

\subsection{When and Why Choose MAS?}

MAS are generally used when the problem is too complex to be solved by a single system because of some software or hardware limitations. In particular, if the component maintains multiple relationships between them. MAS are an excellent tool to ensure an autonomous control system in a widely distributed system and whose characteristics are very dynamic.

For an effective MAS, several agents must work concurrently, which reduces the resolution time considering the used speed which is due to parallelism [19] [20].

When we need a system which must adapt dynamically when adding new components (these components should easily adapt when the environment undergoes modifications); MAS are probably the ideal solution for this kind of scenarios. It should be remembered that one of the most important advantages of 
MAS is their modularity that allows making programming easier, i.e. the addition of new agents to the MAS is not a problem which explains their scalability.

The interest of the agent-based solutions lies in the complete absence of central entity governing the operation of agents, which ensures high strength and high reliability (because if an agent fails, the system continues to function).

\subsection{Interactions between Agents}

One of the main properties of the agent in MAS is interacting with the other agents. These interactions are generally defined as any form of executed action in the system and which causes changing the behavior of another agent.

An interaction is a dynamic linking of two or more agents through a set of reciprocal actions. The interactions are expressed from a series of actions whose consequences exert in return an influence on the future behavior of agents [1].

The interaction can be decomposed into three phases not necessarily sequential:

- Information receipt or the perception of a change,

- Reasoning on other agents from the information acquired,

- A transmitting messages or more actions (action plan) modifying the environment.

Common types of interaction include cooperation (working together to solve a common goal), coordination (organizing problem solving so that harmful interactions are avoided or beneficial interactions are exploited); and negotiation (reaching an agreement acceptable to all parties involved).

\section{$3 \quad$ Multi Agent Systems and Cognitive Radio}

\subsection{MAS and Spectrum Allocation}

The CR offers a balanced solution to the problem of spectrum congestion by giving first priority use to the spectrum owner, then allowing others to use the unused portions of the spectrum. To intelligently manage the CR resources, trading and cooperation algorithms resulting from the field of multi agent are to be exploited in order to ensure a more efficient allocation of spectrum.

In the CR community, we often talk about cooperation between SUs and negotiation between Pus and SUs. Initially we will discuss the second concept (negotiation) assuming that $\mathrm{CR}$ nodes are fixed.

Ferber could make a typology of interaction situations through the main components of the interaction (goals, resources, competences). Interactions of agents in MAS are motivated by the interdependence of agents according to these three dimensions: their goals can be compatible or not; agents can desire resources that others have, an agent $\mathrm{X}$ may have the necessary capacity to an agent $\mathrm{Y}$ for the fulfillment of the action plan of Y. Table 1 summarizes the various possible situations. 
Table 1. Classification of interaction situations

\begin{tabular}{|c|c|c|c|c|}
\hline Goals & Resources & Competences & Type of situation & Remark \\
\hline Compatibles & Sufficient & Sufficient & Independence & $\begin{array}{l}\text { Situation of } \\
\text { indifference }\end{array}$ \\
\hline Compatibles & Sufficient & Insufficient & Simple collaboration & \multirow{3}{*}{$\begin{array}{l}\text { Situation of } \\
\text { cooperation }\end{array}$} \\
\hline Compatibles & Insufficient & Sufficient & Congestion & \\
\hline Compatibles & Insufficient & Insufficient & $\begin{array}{l}\text { Coordinated } \\
\text { collaboration }\end{array}$ & \\
\hline Incompatibles & Sufficient & Sufficient & $\begin{array}{l}\text { Pure individual } \\
\text { competition }\end{array}$ & \multirow{4}{*}{$\begin{array}{l}\text { Situation of } \\
\text { antagonism }\end{array}$} \\
\hline Incompatibles & Sufficient & Insufficient & $\begin{array}{l}\text { Pure collective } \\
\text { competition }\end{array}$ & \\
\hline Incompatibles & Insufficient & Sufficient & $\begin{array}{l}\text { Individual conflicts } \\
\text { for resources }\end{array}$ & \\
\hline Incompatibles & Insufficient & Insufficient & $\begin{array}{l}\text { Collective conflicts } \\
\text { for resources }\end{array}$ & \\
\hline
\end{tabular}

According to Ferber, the goals are incompatibles if the satisfaction of one causes the dissatisfaction of the other.

In the case of CR, SUs seek to satisfy their application by seeking a free channel and PUs have the opportunity to share their spectrum too. So we can say that the goals are compatibles because there is no contradiction between PUs and SUs goals.

When we speak about resources, we mean the number of available channels (free parts of the spectrum).

In the scenarios we will process, we assume that SUs have sufficient competences, which means that each agent can make the sensing alone (no need to other agents). Based on the classification of interaction situations (Table1), we modeled the scenario that will be encountered in the context of CR through a binary tree in Fig.1.

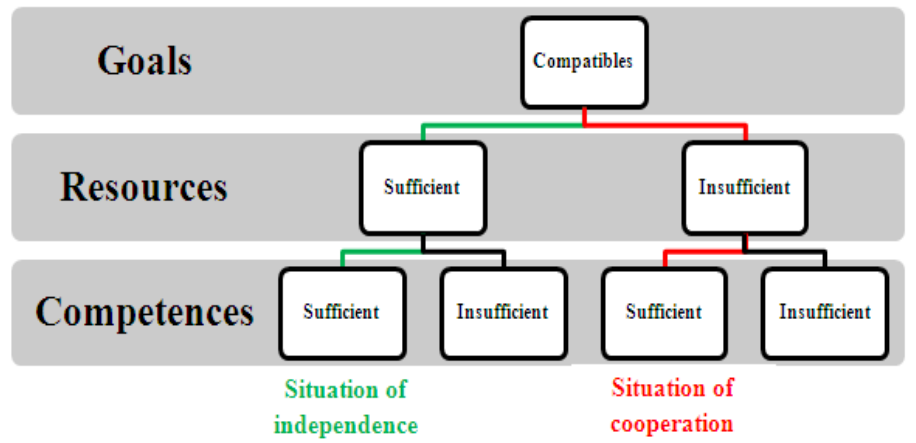

Fig. 1. Binary tree modeling the interactions between agents in the case of $\mathrm{CR}$

In the situation of independence, there is no problem to solve regarding to the interaction of agents because resources and competences are sufficient. This is why we are particularly interested by the situation of cooperation. The goal of researches carried out in the field of cooperation and negotiation between agents is to 
achieve an overall state of MAS by promoting agents synergy. Thus the objective may be to achieve a better state, to improve the overall result while satisfying all the local results.

When the resources used by the agents are limited and they are in a situation of congestion, we use most often:

- The law of the strongest (define a priority according to the strength of the agent), but in the case of CR, SUs have the same goal and want to satisfy their need in spectrum. So setting priorities in this case, returns to favor some types of applications.

- Techniques of negotiation, i.e. compromises will be established between the agents. Indeed, it is interesting to use this method because the installation of these mechanisms would make it possible to lead to acceptance by an agent to cooperate with other agents. In the case of $\mathrm{CR}$, we must only verify whether the PU is ready to cooperate or not.

Subsequently, we will use this method (negotiation) to solve the problem of congestion between SUs.

\subsection{Negotiation Protocols}

A negotiation protocol is the set of rules which direct the interaction. This includes the types of allowed participants, the negotiation states, the events which pass from a state to another and the acceptable and valid actions from participants. In the literature, there are several trading protocols; Table 2 summarizes the most important protocols.

Table 2. Negotiation protocols

\begin{tabular}{|c|l|}
\hline Negotiation protocol & Description \\
\hline Contract Net & $\begin{array}{l}\text { Agents coordinate their activities through the establishment of } \\
\text { contracts to achieve specific goals. }\end{array}$ \\
\hline Auctions theory & $\begin{array}{l}\text { The term "auction" means any technique of establishing a sales } \\
\text { competition, which aims to determine the future owner of the } \\
\text { article concerned in successive bids. }\end{array}$ \\
\hline Heuristic negotiation & $\begin{array}{l}\text { The agents must provide useful reactions for the proposals they } \\
\text { receive, these reactions can take the form of a criticism or a } \\
\text { counter-proposal (refused or modified proposal). }\end{array}$ \\
\hline $\begin{array}{l}\text { Negotiation by } \\
\text { argumentation }\end{array}$ & $\begin{array}{l}\text { An agent may try to persuade another agent to respond favorably } \\
\text { to his proposal by looking for arguments that identify new } \\
\text { opportunities, create new opportunities or changing the evaluation } \\
\text { criteria. }\end{array}$ \\
\hline
\end{tabular}




\subsection{Protocol Choice}

To solve the problem of congestion caused by the lack of resources, and well model the negotiation, a protocol must be selected among those mentioned before. We chose a protocol based on auctions theory because we believe that this is an ingenious approach to allocate resources to a set of agents. It should be understood that the allocation is a difficult problem to the extent that resources are limited compared to the number of requests.

Since an auction restricts negotiating variables to a reduced number of parameters essentially price, this makes it easier for programmers. Finally, an auction leads to a mutually acceptable solution for the seller and buyers (in our case the PU and SUs), markets forces being the only referee of the outcome of the negotiation.

The object of the negotiations in our scenario is the number of free channels proposed by the PU. Initially, we are interested only to the different methods to be used for negotiation between the PU and SUs. These methods will be implemented on the PU side. Interactions between PU and SU will be simulated later using the MAS.

\section{$4 \quad$ Auctions and Cognitive Radio}

\subsection{Types of Auctions}

Currently, there are several auction protocols, only the most frequently used are listed in Fig.2. below.

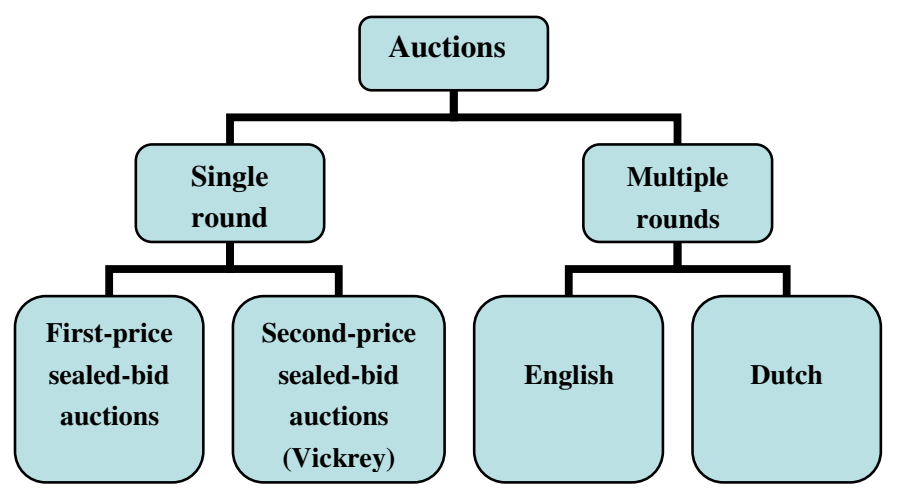

Fig. 2. Organigram showing types of auctions

\subsection{Related Works}

Generally, an auction consists of several stakeholders; Table. 3 describes the difference between traditional auctions and what corresponds to each speaker when applying this method to the negotiation in CR networks. 
Table 3. Difference between classical auctions and auctions in CR Networks

\begin{tabular}{|l|l|}
\hline Traditional auctions & Auctions in CR networks \\
\hline Objects to sell & Free channels \\
\hline Bidder & Secondary User (SU) \\
\hline Seller & Primary User (PU) \\
\hline Auctioneer & Regulator \\
\hline
\end{tabular}

Auctions are based on the concept of sale and purchase of goods or services. The main purpose of the use of auctions in CR networks is to provide motivation for SUs to maximize their use of spectrum. To fully utilize the spectrum, dynamic spectrum allocation using auctions has become a promising approach that allows users to rent unused channels by PUs.

In general, the proposed solutions by the different authors working on auctions theory for dynamic spectrum access are based on architecture with infrastructure [7].

In [8], the authors propose a mechanism for an efficient and equitable sharing of spectrum resources where we need a coordinator to manage the operation and model spectrum access in CR networks such repeated auction.

In solutions based on auctions, each channel is assigned to a single network, i.e. there is no notion of SU and $\mathrm{PU}$ in the same channel. In the literature, two possibilities are offered:

- Either the regulator allocates channels to PU; they independently allocate unused portions of their channel for SUs [9].

- Either the regulator allocates the right to be SU or PU in the channel [10].

The method of payment is often a major problem when we want to apply auctions in telecommunications networks; this is why some researchers are trying to find adequate solutions. For example the authors in [11] use second price auctions to solve the problem of spectrum allocation and develop an approach which introduces the concept of fictive money for the payment in real time. Another interesting approach is proposed in [12] where the authors think that there is no concept of money for the auction but the price to pay is the waiting time.

However, some researches has been done by [13] and offer a traditional approach based on auctions, and then they do an extension of their approach to a scenario that assumes that there are free unused channels. i.e. the SU will have the choice between paying a good QoS or access to an unused channel for free and encounter risk of interference with the users (if several SU operate simultaneously on these bands). 
Another way to use auctions is proposed in [14], where the authors have shown that in some scenarios the spectrum is used efficiently when multiple SU gain access to a single channel, this is what distinguish their method with the traditional auctions where only one user can win.

In these solutions, user behaviors can be false, so the centralized manager can't maximize the utility function of the overall network [15].

\subsection{Proposed Network Topology}

Several researchers model the auction as a network with infrastructure; otherwise the regulator is required to conduct the sale.

In this paper, we propose to use network architecture without infrastructure or what is generally called an "ad hoc network", because this type of networks differs from the other forms by its ability to organize itself independently without fixed infrastructure. An ad hoc network consists only of a variable number of entities that communicate with each other directly.

In other words, the communication will be directly done between PUs and SUs. Fig.3 illustrates the network topology that we used:

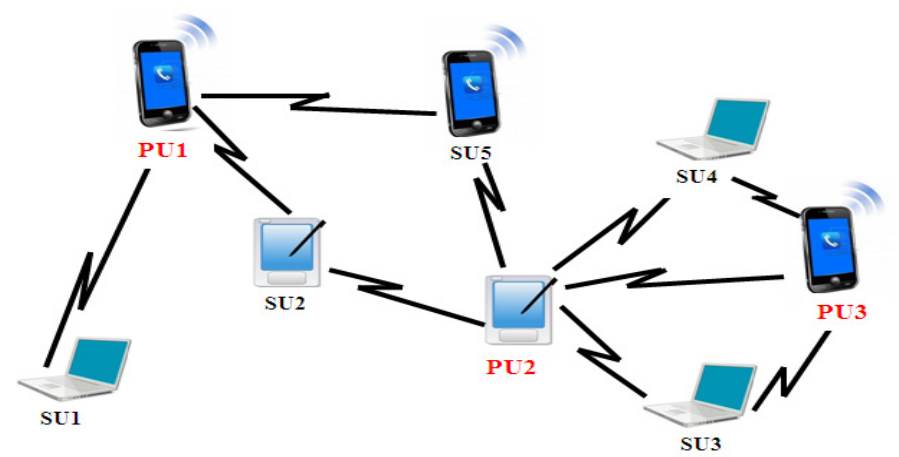

Fig. 3. Network topology (ad hoc mode)

\subsection{Scenario}

Initially, we focused our attention on a particular type of negotiation "one to many" i.e. there is only one PU who shares its spectrum and several SU who need to ensure free channels for assuring the quality of their application. Fig.4 illustrates the scenario that we will deal in the paper.

For example, in Fig.4, there are 3 SUs (SU1, SU2, SU5) who want to access to the free resources at PU1. However there are only 4 free resources at PU1 which is not sufficient to serve the needs of all SUs $(1+2+2=5$ required resources). So which of SU1 or SU2 or SU5 will access to the spectrum? 
To solve this kind of problem, we use dynamic spectrum access techniques, and as mentioned before, we'll use auction theory to manage the spectrum. To be specific, we chose two types of auctions: one taking place in a single round such as First-price sealed-bid auctions and another one taking place in multiple rounds such as English auctions.

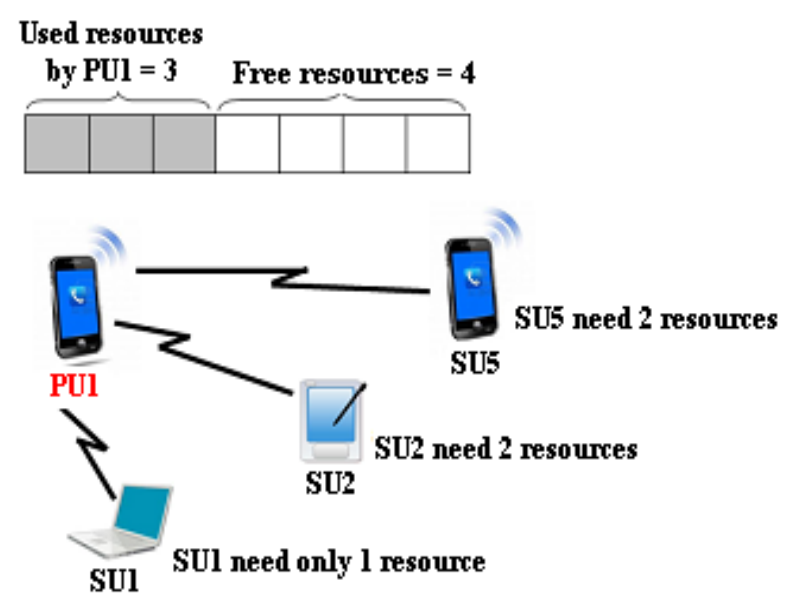

Fig. 4. Proposed scenario

\subsection{Proposed Algorithms}

To solve the spectrum allocation problem, we propose to use dynamic programming which is an algorithmic technique to optimize the amounts of monotonically increasing functions under constraint. This technique applies to optimization problems whose objective function is described as "the sum of monotonically increasing functions of resources."

We note:

$\mathrm{nb}:$ the number of SUs.

$\mathrm{m}$ : the number of free channels at PU.

$\mathrm{W}$ : array of size $\mathrm{nb}, \mathrm{W}[\mathrm{i}]$ is the number of requested channels by SUi.

$\mathrm{C}$ : array of size $\mathrm{nb}, \mathrm{C}$ [i] is the proposed price for $\mathrm{W}$ [i] by $\mathrm{SU}_{\mathrm{i}}$.

The increasing monotonic function to be optimized is: $\operatorname{Max} \sum_{i=0}^{n b-1} C[i]$ 
The constraint is: $\sum_{i=0}^{n b-1} W[i] \leq m$

We therefore proposed and implemented the following algorithms on the PU side.

\subsubsection{For First-Price Sealed-Bid Auctions}

The initiator starts the auction and each participant submits a bid in an envelope or electronically in a single round (turn), without knowing the bids of the others. The participant who made the biggest bid wins the object and pays the amount of its offer. Fig.5. shows the algorithm we have been proposed to solve this type of auction.

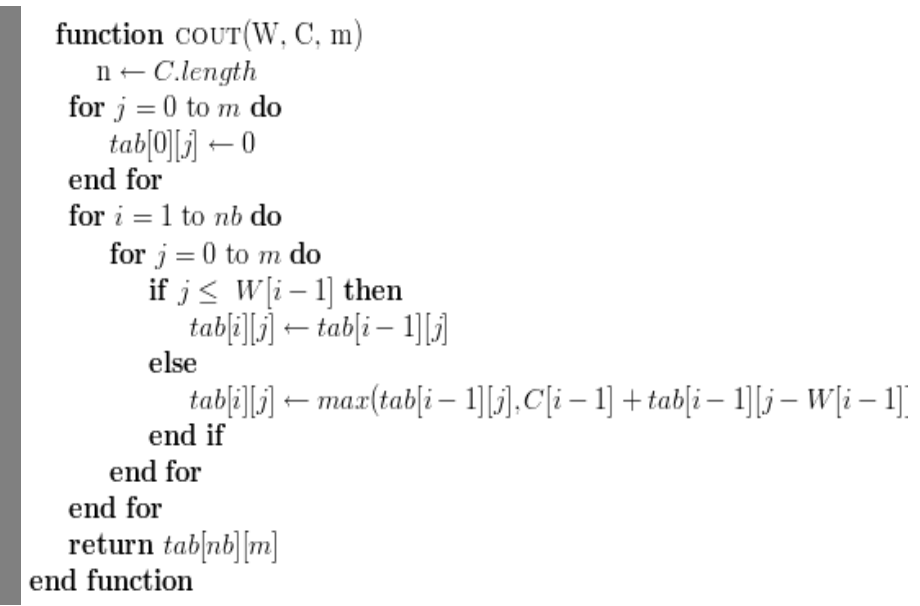

Fig. 5. Algorithm for first-price sealed-bid auctions

\subsubsection{For English Auctions}

The initiator starts usually the auction by announcing a reservation price (the minimum price for which he is willing to sell the item). Each participant announces publicly its offer in several successive rounds. When no participant wants to increase his bid, the auction ends and the participant who made the biggest offer wins the object at its offer price. Fig.6 shows the algorithm that was used to solve this type of auction. 


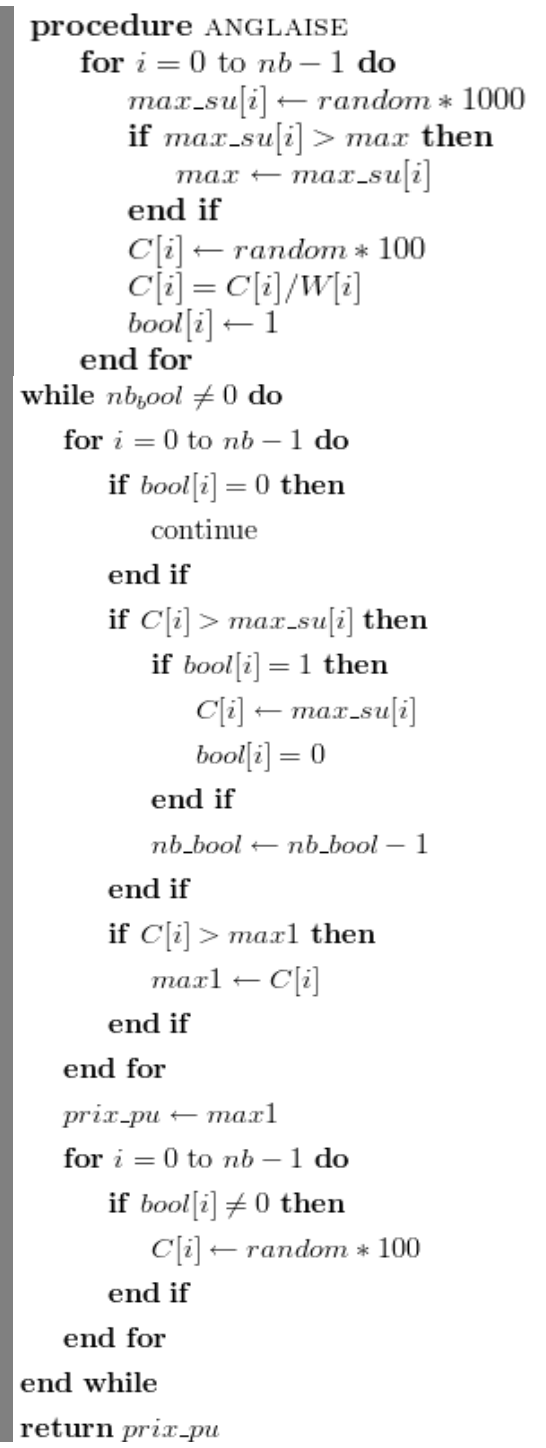

Fig. 6. Algorithm for english auctions

\subsection{Comparison between the Two Types of Auctions}

We implemented both algorithms on the PU and then compared the obtained results knowing that we tested the two algorithms assuming that there is 10 SUs in the same place. 


\subsubsection{Comparison in Terms of Running Time}

La Fig.7 shows that the execution time of multiple rounds auction is very large compared to those taking place in a single round.

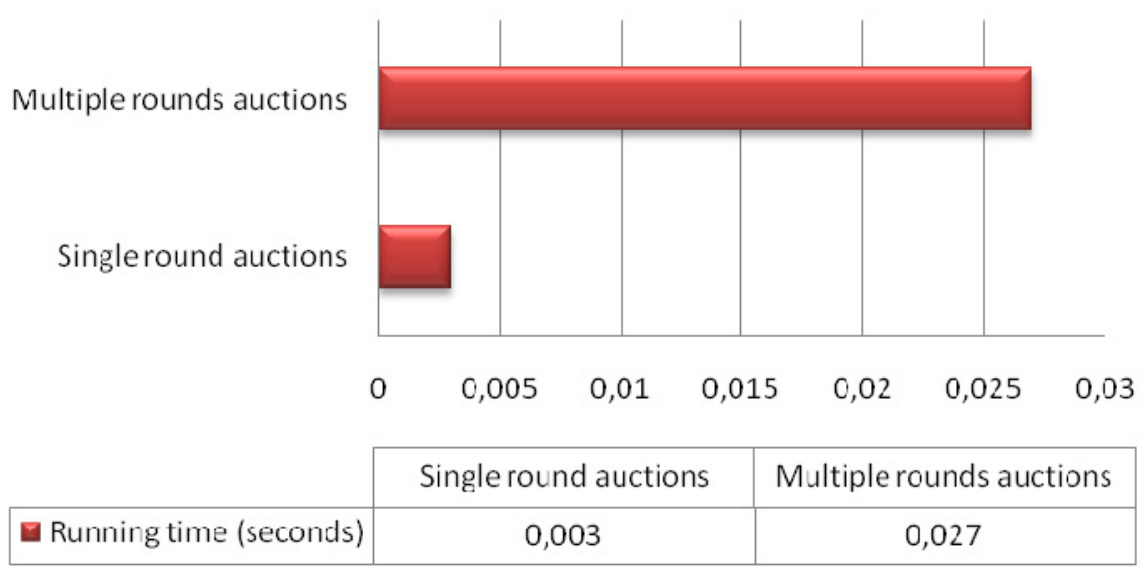

Fig. 7. Comparison between the two algorithms in terms of running time

\subsubsection{Comparison in Terms of Efficiency}

In our case, when we talk about efficiency, we talk about number of satisfied SUs. For this, we compared the two algorithms and we note that the results are the same.

Fig.8 shows the impact of auctions on the number of satisfied SUs. We clearly see that whatever the number of available channels at the PU, the number of satisfied SUs remains the same with both methods (blue and red curves).

\section{Impact of auctions on the number of satisfied SUs}

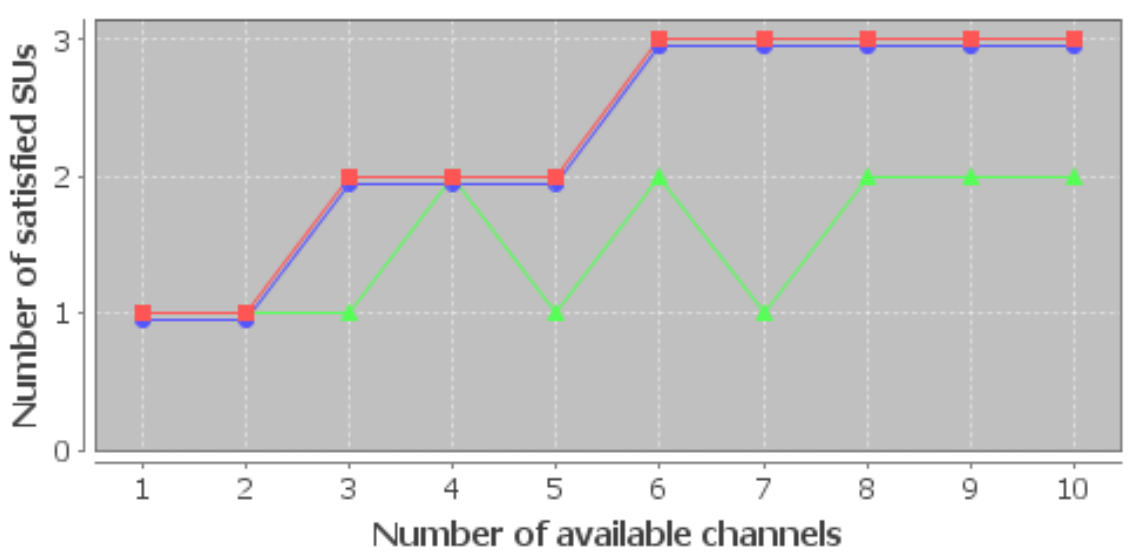

- Single round auctions $\rightarrow-$ Multiple rounds auctions $-\mathbf{t}$ Withour auctions

Fig. 8. Impact of auctions on the number of satisfied SUs 
We compared also the use of auction with the case where we don't use this technique (PU satisfies the first received demand according to his free channels), we notice that the number of satisfied SUs is always higher compared to that obtained without auctions.

The parameters we used are: $\mathrm{nb}=5, \mathrm{C}=\{10,20,40,120,260\}$ and $\mathrm{W}=\{7,5,3,2,1\}$.

To determine the number of SU that can be satisfied in a given period of time $(1 \mathrm{~min}=60 \mathrm{~s})$, we made a comparison between the use of the two previous algorithms (single round auctions and multiple rounds auctions) with the FIFO method (First In First Out).

For this comparison, we assumed that the operating time of each channel is $10 \mathrm{~s}$. In addition to the execution time and operating time, we must also know that the necessary time to exploit a channel (T. establishment) is 5s on average [14] [15].

\section{T. required $=T$. execution $+T$. establishment $+T$. operating}

The dataset we used is: $\mathrm{m}=4, \mathrm{nb}=4, \mathrm{C}=\{44,94,151,97\}$ and $\mathrm{W}=\{3,2,1,3\}$.

Fig.9 shows that in 60s, 3 SUs were satisfied with the use of the two auction methods, however, using the FIFO method only 2 SUs were satisfied.

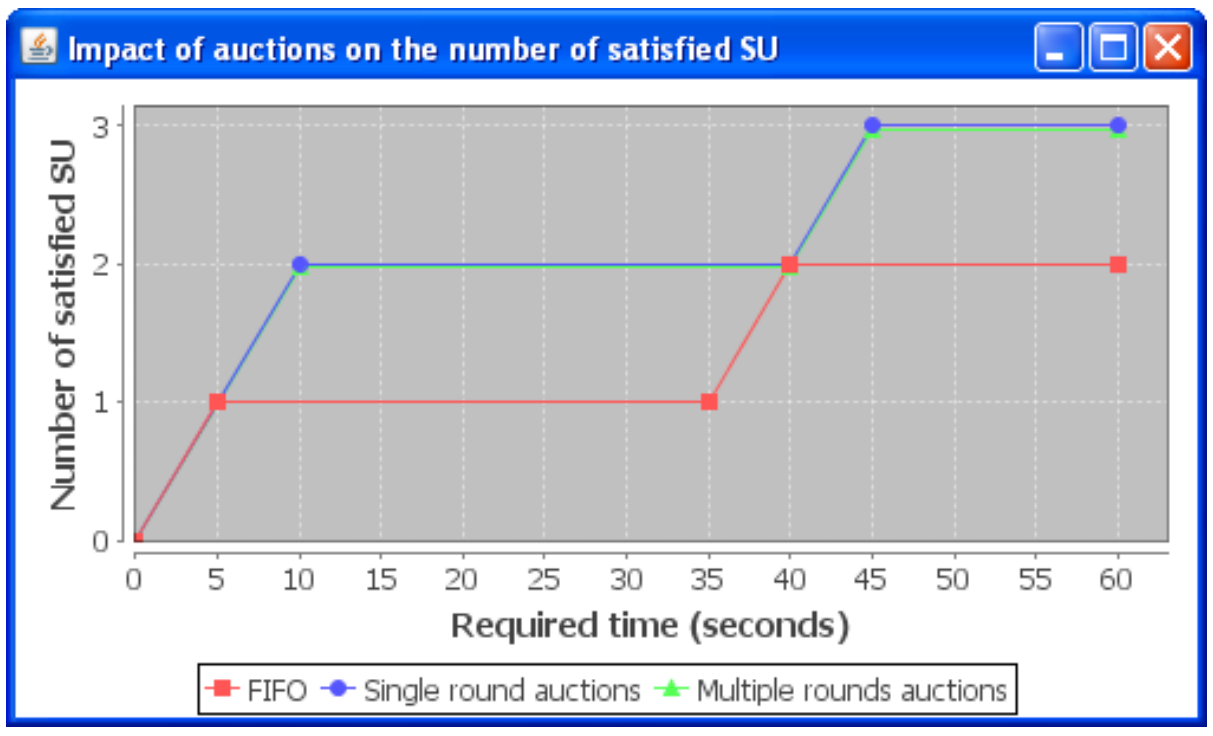

Fig. 9. Impact of auctions on the number of satisfied SUs

Fig.10 below shows the impact of auctions on the gains made by the PU.

The dataset we used is: $\mathrm{nb}=10, \mathrm{C}$ (single round) $=\{39,51,160,59,64,145,177$, $53,42,106\}, \mathrm{C}$ (multiple rounds) $=\{286,209,141,489,3,21,671,226,622,350\}$ and $\{\mathrm{W}=3,2,1,3,2,1,1,2,3,1\}$. 


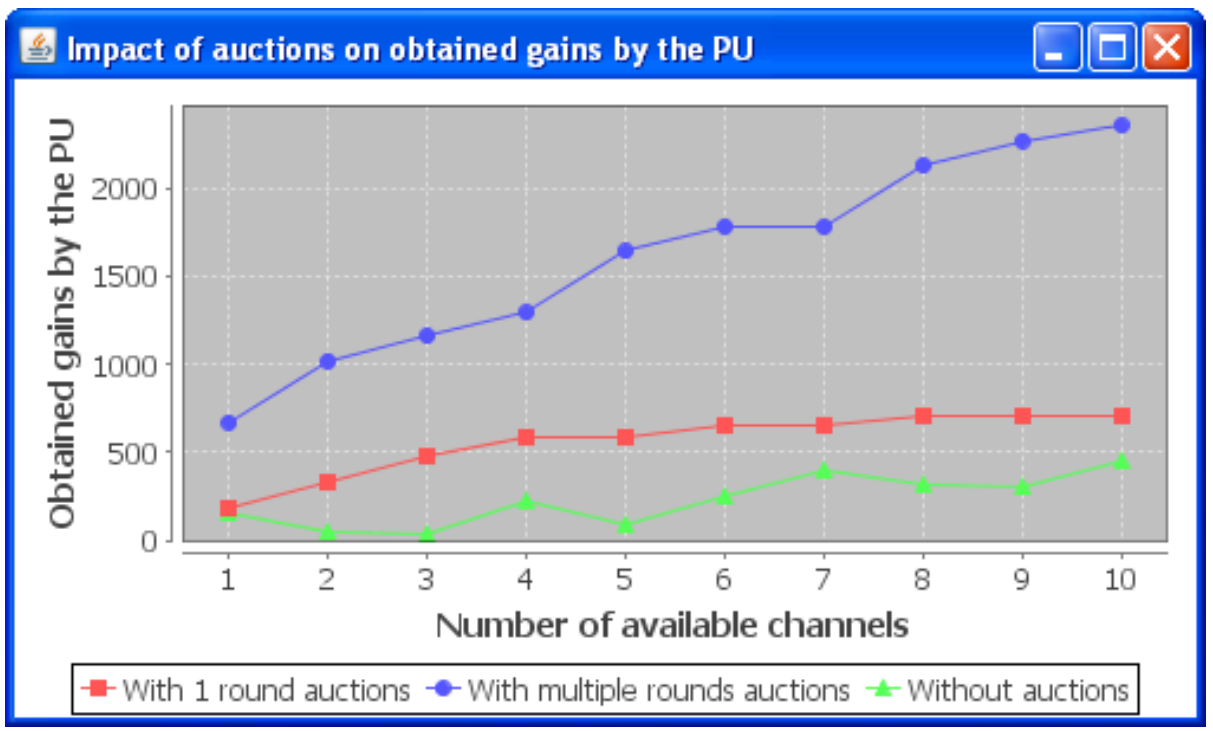

Fig. 10. Impact of auctions on the obtained gains by the PU

We note that the use of multiple rounds auctions is more beneficial for PU because their earnings are much higher compared to the use of single round auctions or the use of the FIFO technique.

Whatever the number of required channels, the SU offers a value for all the requested channels; so it's always the one who can offer the highest value who wins. This is what makes that the efficiency of algorithms remains the same since we satisfy the same number of SUs.

However, the execution time is a very important criterion that can't be neglected because we work on real-time applications, so that the response time should be minimal and we really can't afford to waste time using a method which can take time.

To maximize the gains of the PU, we have to use multiple round auctions. If we are interested in the number of satisfied SU, it is better to use a single round auction because the procedure is faster.

\section{Conclusion}

In the context of $\mathrm{CR}$, negotiation is one of the simplest solutions to address congestion caused by lack of available resources for SU. We think provide broader view of the negotiation by treating the case where there are several PUs (negotiation many to many).

Our approach has proven that it is preferable to use a single round auction especially if we seek to satisfy applications that require an immediate response, because the use of multiple rounds auctions can make us lose a few seconds since the procedure is slightly longer and slower. 
Our method has also proven that to solve the problem of spectrum congestion, we should use one of dynamic spectrum access techniques rather than using nothing and satisfy the first received request.

In our future works, we think we can improve the reliability of wireless links and ensure good QoS to CR mobile terminals [16] [17] [18] by integrating Multi Agent Systems.

\section{References}

1. Amraoui, A., Benmammar, B., Bendimerad, F.T.: Accès Dynamique au Spectre dans le Contexte de la Radio Cognitive. In: 2ième édition de la conférence nationale de l'informatique (JEESI 2012), ESI, Oued-Smar (Alger), Algérie (Avril 2012)

2. Ferber, J.: Les systèmes multi-agents. In: Vers une intelligence collective. InterEditions, Paris (1995)

3. Jennings, N.R.: Agent-Oriented Software Engineering. In: Garijo, F.J., Boman, M. (eds.) MAAMAW 1999. LNCS, vol. 1647, pp. 1-7. Springer, Heidelberg (1999)

4. Casteran, J.C., Gleize, M.P.: Des méthodologies orientées multi-agent. In: Hermes, D. (ed.) Actes des Journées Francophones sur les l'Intelligence Artificielle Distribuée et les Systèmes Multi-Agents, JFIADSMA 2000, pp. 191-207 (2000)

5. Chang, H.-B., Chen, K.-C., Prasad, N.R., Su, C.-W.: Auction Based Spectrum Management of Cognitive Radio Networks. IEEE Transactions on Vehicular Technology, 1923-1935 (May 2010)

6. Han, Z., Zheng, R., Poor, H.V.: Repeated Auctions with learning for Spectrum Access in Cognitive Radio Networks. In: Allerton Conference on Communication, Control, and Computing 2009 (2009)

7. Ji, Z., Ray Liu, K.J.: Belief-Assisted Pricing for Dynamic Spectrum Allocation in Wireless Networks with Selfish Users. In: Proc. of IEEE SECON (2006)

8. Kasbekar, G.S., Sarkar, S.: Spectrum Auction Framework for Access allocation in Cognitive Radio Networks. In: MobiHoc (2009)

9. Chen, B., Wu, H.-K., Hoang, A.T., Liang, Y.-C.: Optimizing the second-price auction algorithm in a Dynamic Cognitive Radio Network. In: 11th IEEE Singapore International Conference on Communication Systems, ICCS 2008 (2008)

10. Wu, G., Ren, P., Zhang, C.: A waiting-time Auction Based Dynamic Spectrum allocation in cognitive radio networks. In: GLOBECOM (2011)

11. Chen, L., Iellamo, S., Coupechoux, M., Godlewski, P.: An auction Framework for Spectrum Allocation with Interference Constraint in Cognitive Radio Networks. In: INFOCOM 2010 Proceedings of the 29th Conference on Information Communications (2010)

12. Yongle, W., Wang, B., Liu, K.J.R., Clancy, T.C.: Collusion-resistant multi-winner spectrum auction for cognitive radio networks. In: Proceedings of IEEE GLOBECOM, pp. $1-5(2008)$

13. Mir, U.: Utilization of Cooperative Multiagent Systems for Spectrum Sharing in Cognitive Radio Networks. Thèse 2011 (2011)

14. Busanelli, S., Martalõ, M., Ferrari, G., Spigoni, G.: Vertical Handover between WiFi and UMTS Networks: Experimental Performance Analysis. International Journal of Energy, Information and Communications 2(1) (February 2011) 
15. Daia, Z., Fracchiaa, R., Gosteaub, J., Pellatia, P., Viviera, G.: Vertical handover criteria and algorithm in IEEE 802.11 and 802.16 hybrid networks. In: Laboratoire de Motorola Paris

16. Benmammar, B., Amraoui, A., Baghli, W.: Performance improvement of wireless link reliability in the context of cognitive radio. IJCSNS International Journal of Computer Science and Network Security 12(1), 15-22 (2012)

17. Amraoui, A., Benidris, F.Z., Benmammar, B., Krief, F., Bendimerad, F.T.: Toward cognitive radio resource management based on multi-agent systems for improvement of real-time application performance. In: 5th IFIP International Conference on New Technologies, Mobility and Security, NTMS 2012, Istanbul, Turkey (2012)

18. Amraoui, A., Baghli, W., Benmammar, B.: Improving video conferencing application quality for a mobile terminal through cognitive radio. In: Proceedings of the 14th IEEE International Conference on Communication Technology, ICCT 2012, November 9-11, Chengdu, China (2012)

19. Benmammar, B., Krief, F.: La Technologie Agent et les Réseaux Sans Fil. In: Congrès Des Nouvelles Architectures pour les Communications, DNAC 2003, Paris, France (Octobre 2003)

20. Jrad, Z., Krief, F., Benmammar, B.: An Intelligent User Interface for the Dynamic Negotiation of QoS. In: 10th IEEE International Conference on Telecommunications, ICT 2003, Papeete, Tahiti (February 2003) 